Article

\title{
An Algorithm for the Broad Evaluation of Potential Matches between Actuator Concepts and Heavy-Duty Mobile Applications ${ }^{\dagger}$
}

\author{
David Fassbender *(D) and Tatiana Minav (iD \\ Faculty of Engineering and Natural Sciences, IHA-Innovative Hydraulics and Automation, Tampere University, \\ 33720 Tampere, Finland; tatiana.minav@tuni.fi \\ * Correspondence: david.fassbender@tuni.fi \\ + This paper represents an extension of the work previously presented at the 1st International Electronic \\ Conference on Actuator Technology 2020 under the title "Finding the Perfect Match: Different Heavy-Duty \\ Mobile Applications Call for Different Actuators".
}

check for updates

Citation: Fassbender, D.; Minav, T. An Algorithm for the Broad Evaluation of Potential Matches between Actuator Concepts and Heavy-Duty Mobile Applications. Actuators 2021, 10, 111.

https://doi.org/10.3390/act10060111

Academic Editor: Ioan Ursu

Received: 6 May 2021

Accepted: 19 May 2021

Published: 25 May 2021

Publisher's Note: MDPI stays neutral with regard to jurisdictional claims in published maps and institutional affiliations.

Copyright: (c) 2021 by the authors. Licensee MDPI, Basel, Switzerland. This article is an open access article distributed under the terms and conditions of the Creative Commons Attribution (CC BY) license (https:/ / creativecommons.org/licenses/by/ $4.0 /)$.

\begin{abstract}
In recent years, a variety of novel actuator concepts for the implements of heavy-duty mobile machines (HDMMs) has been proposed by industry and academia. Mostly, novel concepts aim at improving the typically low energy efficiency of state-of-the-art hydraulic valve-controlled actuators. However, besides energy-efficiency, many aspects that are crucial for a successful concept integration are often neglected in studies. Furthermore, most of the time, a specific HDMM is focused as an application while other HDMM types can show very different properties that might make a novel concept less suitable. In order to take more aspects and HDMM types into account when evaluating actuator concepts, this paper proposes a novel evaluation algorithm, which calculates so-called mismatch values for each potential actuator-application match, based on different problem aspects that can indicate a potential mismatch between a certain actuator concept and an HDMM. The lower the mismatch value, which depends on actuator characteristics as well as HDMM attributes, the more potential is the match. At the same time, the modular nature of the algorithm allows to evaluate a large number of possible matches at once, with low effort. For the performance demonstration of the algorithm, 36 potential matches formed out of six actuator concepts and six HDMM types are exemplarily evaluated. The resulting actuator concept ratings for the six different HDMMs are in line with general reasoning and confirm that the evaluation algorithm is a powerful tool to get a first, quick overview of a large solution space of actuator-HDMM matches. However, analyzing the limitations of the algorithm also shows that it cannot replace conventional requirements engineering and simulation studies if detailed and reliable results are required.
\end{abstract}

Keywords: hydraulic actuators; heavy-duty mobile machines; electro-hydraulics; design process; energy efficiency

\section{Introduction}

Next to driving, the main function of a HDMM is fulfilling a work task with its implements. Those implements comprise mostly linear actuators with requirements such as robustness, low price, high safety and compactness. So far, centralized, valve-controlled hydraulic systems have been the standard solution to meet those requirements. Examples of valve-controlled concepts are load sensing (LS) systems, negative flow control or opencenter systems [1], and they all have one major issue in common which is high metering losses caused by the control valves. Due to arising and tightening emission regulations in many countries worldwide, these losses and the related low energy efficiency became more problematic. In response, numerous new actuator concepts have been proposed by researchers over the last decades as replacements for conventional valve-controlled actuators on HDMMs. Major concepts are independent metering [2], multi-pressure rail 
systems [3], displacement control [4], electro-hydraulic actuators (EHAs) [5], hydraulictransformer-based systems [6], digital hydraulics [7] and even non-hydraulic electromechanical actuators (EMAs) [8]. Moreover, Ketelsen et al. could define further 8 subclasses just for EHAs themselves [5].

Figure 1 is depicting the effect of this flood of available concepts nowadays: For each HDMM, a considerably large number of potentially matching actuator concepts with different characteristics and capabilities must be considered in the process of designing a new machine. On the other hand, for the actuator concepts, it is crucial to understand that each HDMM type, depicted on the right side, shows its own application requirements and conditions. Between different HDMMs, attributes such as size, power level, the typical working environment, or the number of actuators can vary a lot. Accordingly, it is unlikely that one actuator concept can be an one-fits-all solution, and each potential actuatorHDMM match —of which plenty exist already in Figure 1, represented by red lines-must be analyzed separately.

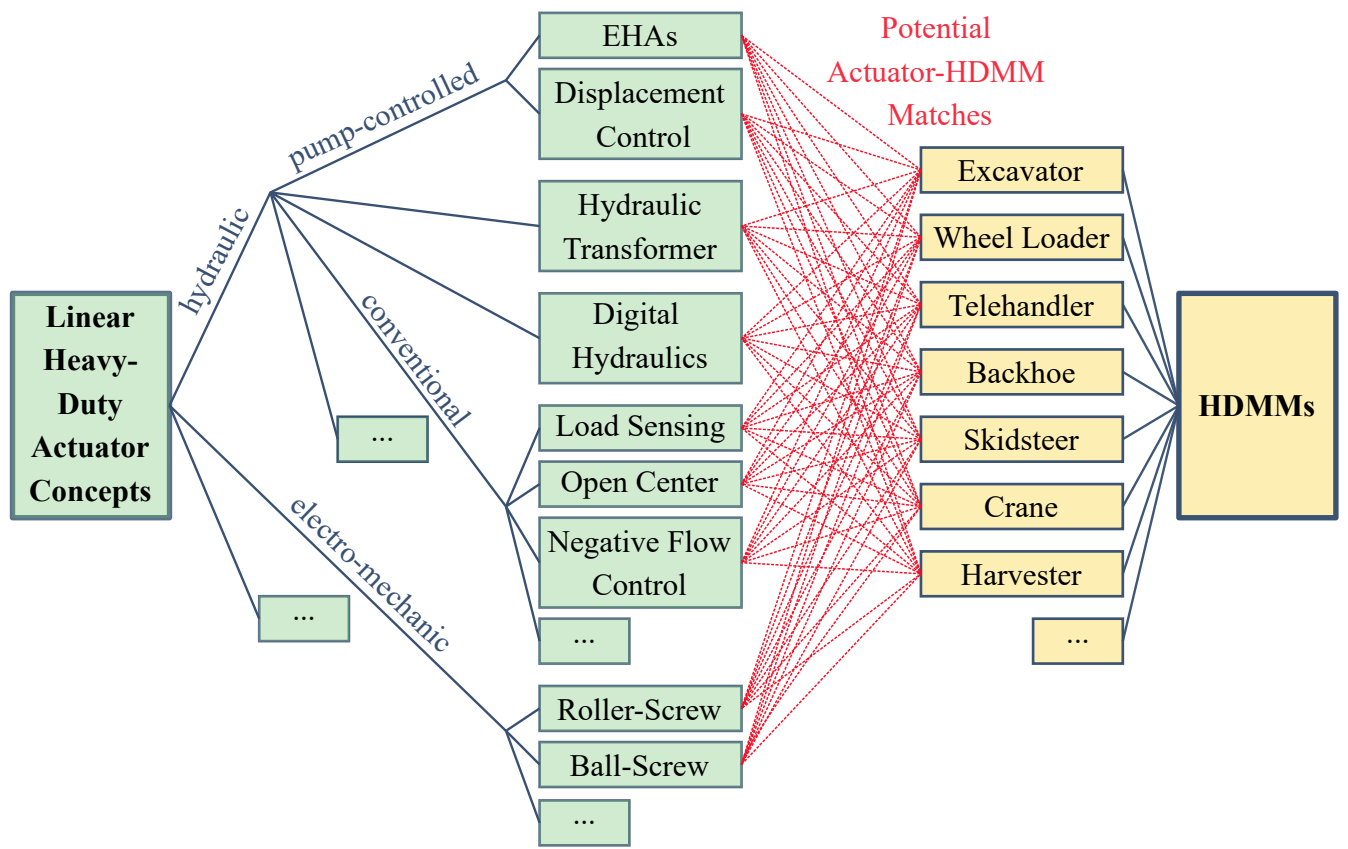

Figure 1. A rough, incomplete classification of linear heavy-duty actuator concepts and of HDMMs as well as the visualization of the resulting potential actuator-HDMM matches.

This paper presents a method that allows to evaluate such a large set of potential matches with low effort in order to get general insights such as:

- Which actuator concept is most promising for a certain machine type?

- Which actuator concept is performing strong in general?

- Are there actuator concepts that are generally less promising but can perform very well for a special machine type?

Design theories and also operations research (OR) are dealing with solving similar issues. However, traditional design tools such as evaluation matrices can easily fail here since a separate evaluation matrix had to be created and analysed for each match, which would be too time consuming. Furthermore, the facts that the evaluation of actuatormachine matches is multidimensional, that properties of different machines can vary a lot, and that operation conditions can change or be even unknown, make it hard to use simulation or experimental approaches in order to formulate general findings-even if only one specific match is focused. One actuator concept might perform very well on a machine for a specific work task but only weakly for another task, for example. Similar problems that are hard to handle due to complexity, uncertainty or dynamic changes-often of social nature-are known as wicked problems [9] in OR. 
Inspired by the way that wicked problems can be handled, this paper-as an extension of the work previously published by the authors in [10]—chooses similar approaches for the technical evaluation of actuator-machine matches. Instead of doing simulations or calculations of specific matches with limited meaning for other matches, a large solution space-meaning many matches-is analysed at once. The focus is on comparing different matches with each other rather than giving absolute statements on how promising a single match is. Furthermore, the nature of the problem allows to classify the actuator concepts and HDMM types giving them attributes that can be quantified and mathematically processed. This makes the evaluation highly modular and allows to adapt the evaluation to changing conditions or requirements. Moreover, the mathematical character allows stakeholders to adjust parameters and understand the influence of certain aspects on the evaluation results. To the authors' knowledge, this is the first time that such an adaptable and comprehensive tool for the evaluation of many different HDMM-actuator combinations is presented.

The article is structured in the following way: Firstly, the numerical concept of the novel match evaluation algorithm is introduced for the specific example of HDMMs and related actuator concepts. In a second step, six representative actuator concepts are prepared to be evaluated by the algorithm. Similarly, the paper also rates different representative HDMM types as counterparts for the evaluation. In a next section, 36 potential matches formed out of the six actuator concepts and six HDMM types are exemplarily evaluated, and the results as well as the algorithm itself are discussed. Finally, limitations of the algorithm are listed and addressed, followed by a conclusion of the article.

\section{Concept for the Machine-Actuator-Match Evaluation}

\subsection{Defining the Differences between a Good and a Bad Match}

Understanding the fundamental criteria that make up a good match between an actuator concept and its application is the basis for developing an algorithm that can evaluate whether an arbitrary combination of an actuator concept and an application is a potential match or not. For this purpose, the following perspective is taken: The quality of an actuator-application match is low if certain problem aspects appear and comparably high if some or all of those problem aspects can be avoided or reduced. "Low energy efficiency" could be one of those problem aspects as it leads to emissions and high energy costs. An actuator that has the corresponding characteristic "causing high energy losses", such as a valve-controlled actuator, tends to be a worse match for an HDMM than a novel actuator concept with the characteristic "causing low energy losses" that reduces the problem aspect "low energy efficiency".

However, there is more than a single problem aspect that must be considered in order to obtain a holistic statement about the match quality. "High component costs per actuator" could be a second problem aspect for the match evaluation. Concerning this aspect, the novel actuator concept might be more problematic than the conventional valve-controlled actuator. This means that every actuator concept can have characteristics that improve certain problem aspects while other characteristics worsen other problem aspects. Crucial is the balance between the overall problem avoidance and the problem worsening. The actuator concept with the most positive balance among other concepts forms the best match.

Moreover, the significance of each problem aspect and its influence on the balance depend highly on the attributes of the concerning HDMM application. While the aspect "low energy efficiency" is more problematic for an HDMM that has a high energy turnover, it might be of lesser importance for a machine with low energy consumption, but the aspect "high component costs per actuator", on the other hand, might be even more problematic because that machine comprises particularly many actuators.

The best actuator-HDMM match is determined by being least problematic concerning a set of problem aspects. This can be identified by looking at the problematic actuator characteristics concerning each problem aspect and the HDMM attributes that define how significant each problem aspect is. 


\subsection{Evaluation Algorithm}

After setting up this general definition, it is step-by-step numerically applied for the match evaluation:

(1) For each multi-dimensional actuator-HDMM-match evaluation, $n \in \mathbb{N}$ problem aspects are considered in order to account for all essential match dimensions.

(2) For each problem aspect $i \in[1, n]$, a problematic actuator characteristic and an HDMM attribute exist.

(3) Every actuator concept receives numbers $c_{i} \in[0,5]$ where 0 means the problematic actuator characteristic for problem aspect $i$ is "not applying" and 5 it is "strongly applying".

(4) For each HDMM type, the numbers $a_{i} \in[0,5]$ mean that the HDMM attribute for problem aspect $i$ is 0 "not expressed" to 5 "strongly expressed".

(5) If $c_{i}$ and $a_{i}$ are multiplied, the problem degree of the match concerning problem aspect $i$ is obtained.

(6) Summing up the problem degrees for all $n$ aspects, the so-called mismatch value $(m m v)$ results.

(7) Finally, among multiple possible matches, the match with the highest mismatch value is the worst, and the match with the lowest mismatch value is the most promising match.

For convenient handling, the evaluation concept is expressed as a vector operation in Equation (1). The mismatch value ( $m m v$ ) is calculated by the scalar product of an HDMMattribute vector $\boldsymbol{a}$ and an actuator-characteristics vector $\boldsymbol{c}$. The vector $\boldsymbol{a}$ contains HDMM attribute expressions for each of the $n$ problem aspect and the vector $c$ the $n$ problematic actuator characteristics respectively. Due to the nature of the scalar product, the result equals the sum of all $n$ problem degrees.

$$
m m v=\boldsymbol{a} \cdot \boldsymbol{c}=\left(\begin{array}{c}
a_{1} \\
a_{2} \\
\vdots \\
a_{n}
\end{array}\right) \cdot\left(\begin{array}{c}
c_{1} \\
c_{2} \\
\vdots \\
c_{n}
\end{array}\right)=a_{1} \cdot c_{1}+a_{2} \cdot c_{2}+\ldots+a_{n} \cdot c_{n}
$$

\subsubsection{Considered Match Aspects}

The selection of problem aspects for the match evaluation algorithm has a strong impact on the quality of the evaluation outcome. If significant problems that are likely to occur with certain actuator concepts are neglected, those concepts would be favored by the algorithm and rated too good. On the other hand, if many insignificant aspects are included as problem aspects, they can outweigh more critical problem aspects and shift the evaluation results. Accordingly, the right set of aspects depends on the types of actuator concepts that are to be evaluated and should include all the potential problem aspects that are related to those but no other. Identifying those requires expert knowledge of the actuator concepts but also of the HDMMs that are supposed to be evaluated since for each aspect a problematic actuator characteristic and an HDMM attribute need to be given. Ideally, this is done by multiple experienced engineers. The set of problem aspects for the evaluation in this paper can be seen in Table 1. The problematic nature of those aspects for certain actuator concepts was identified through literature review and personal experience of the authors; it will become more apparent in the following analysis section (Section 3). 
Table 1. Considered problem aspects for the match evaluation.

\begin{tabular}{|c|c|c|c|}
\hline$i$ & Problem Aspect & HDMM Attribute $\left(a_{i}\right)$ & $\begin{array}{l}\text { Problematic Actuator } \\
\text { Characteristic }\left(c_{i}\right)\end{array}$ \\
\hline 1 & $\begin{array}{l}\text { high component costs for } \\
\text { main actuators }\end{array}$ & $\begin{array}{l}\text { high number of actuators with high } \\
\text { energy turnover }\end{array}$ & high price per actuator \\
\hline 2 & $\begin{array}{l}\text { high component costs for } \\
\text { auxiliary actuators }\end{array}$ & $\begin{array}{l}\text { high number of actuators with low } \\
\text { energy turnover }\end{array}$ & high price for auxiliary actuators \\
\hline 3 & achieving large actuator sizes & containing large actuators & issues with larger actuators \\
\hline 4 & power distribution costs & $\begin{array}{l}\text { long distances between actuators and main } \\
\text { body of HDMM }\end{array}$ & high costs per supply-line length \\
\hline 5 & achieving high actuator power & high actuator power requirements & power-upscaling issues \\
\hline 6 & low energy efficiency & $\begin{array}{l}\text { high energy turnover of implements } \\
\text { in general and compared to other machine } \\
\text { functions like driving }\end{array}$ & $\begin{array}{l}\text { low single-actuator } \\
\text { energy efficiency }\end{array}$ \\
\hline 7 & $\begin{array}{l}\text { energy losses due to } \\
\text { simultaneous actuation }\end{array}$ & $\begin{array}{l}\text { high amount of simultaneous } \\
\text { actuator operation }\end{array}$ & $\begin{array}{c}\text { low energy efficiency during } \\
\text { simultaneous operation } \\
\text { of actuators }\end{array}$ \\
\hline 8 & making use of energy recuperation & high amount of load braking & no/low recuperation potential \\
\hline 9 & overheating of components & $\begin{array}{l}\text { potentially medium to high } \\
\text { environment temperatures }\end{array}$ & low cooling capability \\
\hline 10 & space requirements at actuator position & narrow actuator installation space & high actuator volume \\
\hline 11 & $\begin{array}{l}\text { space requirements at main body } \\
\text { of machine }\end{array}$ & limited space on main body of HDMM & $\begin{array}{l}\text { high volume of centralized } \\
\text { actuator supply }\end{array}$ \\
\hline 12 & low payload due to actuator weight & influence of actuator mass on payload & low power density of actuator \\
\hline
\end{tabular}

\subsubsection{Not Scalable Factors}

For certain problem aspects, actuator concepts can also show problematic characteristics that do not solely scale with the extend of the HDMM attribute expression. An example is aspect 1 in Table 1 , which refers to the actuator costs. For many concepts, fixed costs that are independent of the HDMM attribute-number of actuators-exist, such as the costs for a centralized LS supply. In [11], it was shown how this effect influences whether an LS or an EHA approach shows lower component costs depending on the number of actuators. In order to take this phenomenon into account, the algorithm can be extended by fixed problem terms $f_{i}$ if the related aspect has an attribute-independent component. This can be seen in Equation (2) for the first problem aspect.

$$
m m v=\boldsymbol{a} \cdot \boldsymbol{c}+f_{1}
$$

Because the fixed factors $f_{i}$ are not multiplied by any HDMM attribute value, it makes sense to define $f_{i} \in[0,15]$ instead of giving it a value from 0 to 5 ; so it can have a similar influence on the mismatch value as the scalable factors have.

\subsubsection{Weighing Match Aspects Differently}

This extension of the algorithm is introduced in this paper in order to allow giving certain match aspects more weight or importance than others. Therefore, a weight matrix $\boldsymbol{W}$ is applied as shown in Equation (3). The matrix elements $w_{i}$, which are equal to 1 by default, can be increased for more influence of the aspect $i$ or decreased for less influence. For example, if the HDMM is to be used in environments with very strict emission regulations, the elements $w_{5}, w_{6}$ and $w_{7}$ can be increased to give energy efficiency a higher weight. On the other hand, they could be decreased to give efficiency less weight in the case of no emission restrictions and very low energy prices. Furthermore, if a non-scalable factor 
(see Section 2.2.2) is used, it must be multiplied with the weight factor $w_{i}$ as well as can be seen in Equation (3), which also represents the final form of the evaluation algorithm that is going to be applied in Section 5 .

$$
m m v=W \cdot \boldsymbol{a} \cdot \boldsymbol{c}=\left(\begin{array}{cccc}
w_{1} & 0 & \ldots & 0 \\
0 & w_{2} & \ldots & 0 \\
\vdots & \vdots & \ddots & \vdots \\
0 & 0 & \ldots & w_{n}
\end{array}\right) \cdot\left(\begin{array}{c}
a_{1} \\
a_{2} \\
\vdots \\
a_{n}
\end{array}\right) \cdot\left(\begin{array}{c}
c_{1} \\
c_{2} \\
\vdots \\
c_{n}
\end{array}\right)+w_{1} \cdot f_{1}
$$

Furthermore, a weight matrix can be obtained by multiplying $m$ other weight matrices:

$$
\boldsymbol{W}=\prod_{i=1}^{m} \boldsymbol{W}_{i}
$$

This way, weight aspects can be easily combined. For the evaluation in Section 5, a basic weight matrix is defined, which can be combined with two additional weight matrices:

- The basic weight matrix $\boldsymbol{W}_{0}$ tunes the influence ratio of different aspects by giving less important aspects a reduced weight of 0.5. $w_{4}$ is reduced because the costs for supply lines will generally be on a lower level than the costs for the actual actuator components; $w_{10}$ and $w_{11}$ are reduced since these aspects concern the mechanical design of the machine, which can generally be changed in order to allow for larger components if necessary; and $w_{12}$ is decreased since a reduced pay load can easily be compensated by choosing actuators with higher force capability and reinforcing the mechanical structure.

- With the matrix $\boldsymbol{W}_{\text {costs, }}$ an additional emphasis can be put on reducing component costs. For this purpose, the weight factors $w_{1}, w_{2}$ and $w_{4}$ are increased to the value 2 since they all relate to aspects that increase the component costs.

- With the matrix $\boldsymbol{W}_{\text {efficiency, }}$ a focus is put on energy-efficiency. The weights $w_{6}, w_{7}$ and $w_{8}$ relate to efficiency and are increased to the value 2 for this matrix.

\section{Characterization of Heavy-Duty Actuator Concepts}

In this section, six representative actuator concepts for HDMMs are analyzed and their problematic characteristics are rated in the form of a vector $c$ as described in the previous section. In Section 5, these vectors will be used for the demonstration of the evaluation algorithm.

\subsection{Valve-Controlled Load-Sensing Actuators}

Valve-controlled actuators are the current state-of-the-art concept to drive HDMM implements, and the LS concept is among the most common forms of supplies for those actuators. Therefore, the concept is chosen for this paper to represent a state-of-the-art baseline.

The simplified schematics of an LS system in Figure 2 are used to analyze the characteristics vector of this concept (Equation (6)). The LS supply can be formed by a constant-speed motor (e.g., combustion engine) and variable-displacement pump, or a variable-speed motor (e.g., electric) and a fixed-displacement pump, or a combination of both. Furthermore, each actuator requires its own control valve(s). A main advantage is that one LS supply can be used for multiple actuators and only needs to be scaled up for more flow if actuators have to operate simultaneously at high speeds. Moreover, the concept is very mature as well as standardized, and compact valve assemblies are available at low prices. Thus, adding more actuators requires minimal financial effort-no matter if it is a main actuator or an auxiliary actuator-and $c_{1}$ as well as $c_{2}$ are rated low (1). On the other hand, the fixed costs for the LS supply are rather high, which is expressed by

$$
f_{1, L S}=9 \text {. }
$$




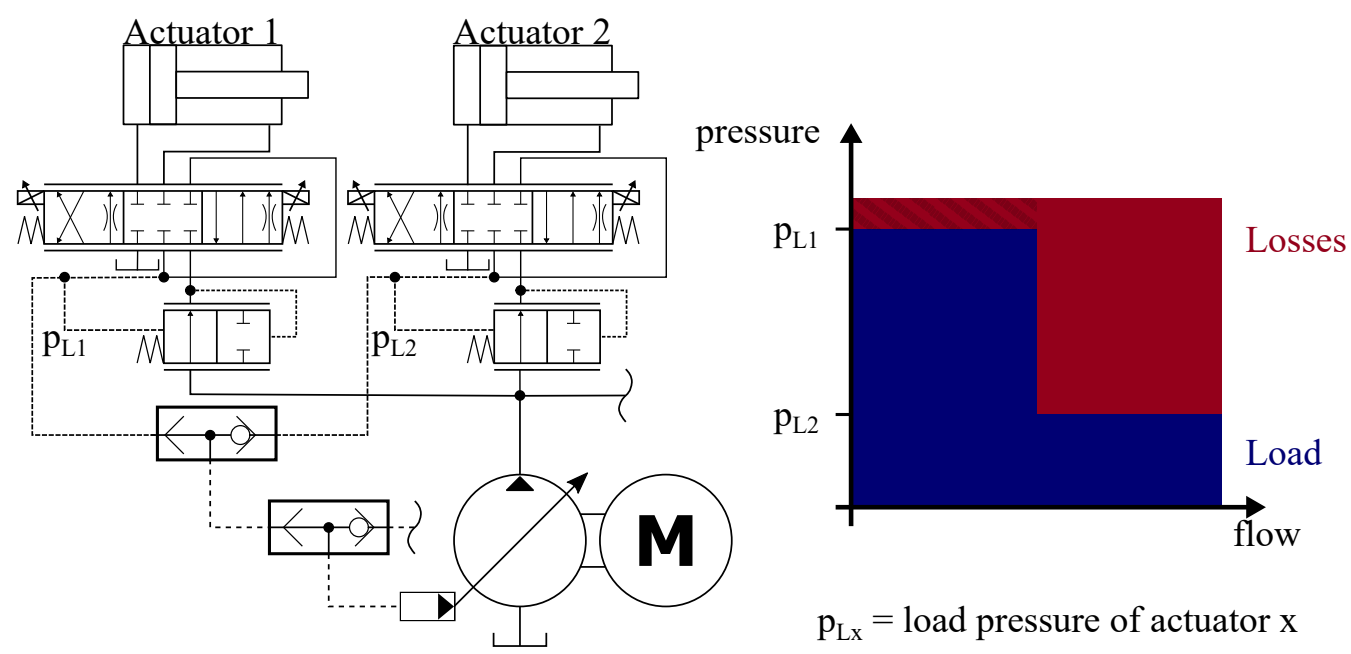

Figure 2. Simplified load sensing system with two actuators (but option for more) and the related pressure-flow diagram for a specific load situation.

Because the size of the actuator only influences the required supply flow, larger actuators can be easily handled by choosing components with higher nominal flow, and $c_{3}$ is rated 1 . Since the system is centralized, expensive hydraulic high-pressure lines need to connect each actuator with the supply, which leads to a high $c_{4}$ (3). For higher power demands, pump and motor can easily be increased in size, and LS systems can often be found on large HDMMs with high power demands. Thus, $c_{5}$ is rated low (1).

In terms of energy efficiency, the pressure-flow diagram in Figure 2 provides more insights. If actuator 1 was operated alone, only the small shaded loss area would be present and the system efficiency relatively high, which is why $c_{6}$ is still rated only medium high (3). Regardless, the efficiency can be extremely low when another actuator with lower load pressure is operated simultaneously such as actuator 2 in Figure 2. In this case, the load pressure difference is compensated by throttling valves, and the power represented by the red area is dissipated into heat. Accordingly, the aspect 7 and factor $c_{7}$ were introduced especially for valve-controlled actuator concepts that generally all have a highly problematic characteristic concerning this aspect. $c_{7}$ is thus rated 3 (not worse because the effect is only significant if the load pressures differ a lot).

Furthermore, braking energy can only be dissipated by valves in this system and not recuperated, which leads to a high $c_{8}$ value (5). However, cooling is easy because the centralized design allows to utilize a common cooler for all actuators together. Thus, $c_{9}$ is rated 2 but not 1 because the cooler still requires extra costs, space and energy.

Concerning the weight and mass of a typical LS actuator it can be said that the actuator itself, a hydraulic cylinder, has a very compact diameter, and even though the principle requires a minimal installation length that is slightly longer than the maximum actuator stroke, the actuator installation length can be rated as low compared to other actuator concepts. For applications with critical installation length, also hydraulic telescope cylinders can be used where the minimal installation length is much shorter than the maximum stroke. Accordingly, the characteristics $c_{10}$ and $c_{12}$ are rated low (1). On the other hand, the centralized LS supply, which also comprises a hydraulic tank and a cooling system next to the pump and motor, requires a significant amount of space somewhere on the machine, and $c_{11}$ is rated 4 .

$$
c_{L S}^{\top}=\left(\begin{array}{llllllllllll}
1 & 1 & 1 & 3 & 1 & 3 & 3 & 5 & 2 & 1 & 4 & 1
\end{array}\right)
$$

3.2. Load-Sensing System with Electric-Generator-Hydraulic-Motor Units for Higher Efficiency of Single Actuators

This concept, presented by the authors in [12], was developed with the target to improve the efficiency of conventional LS systems while keeping up their strengths. It is 
part of the evaluation in this paper in order to show how significant the improvement tends to be for different HDMM applications. The main idea of the concept is to use a combination of electric generator and hydraulic motor (EGHM) for the highly efficient control of actuator functions with high energy turnover and recuperation potential, but conventional LS control valves for actuator functions with low energy turnover where increased component costs cannot be justified. Both actuator types are connected to and benefit from the same LS supply pump as can be seen in the schematics in Figure3. For a detailed concept explanation, the authors refer to [12]; here, only the aspects that define the values for the characteristics vector (Equation (8)) are discussed.

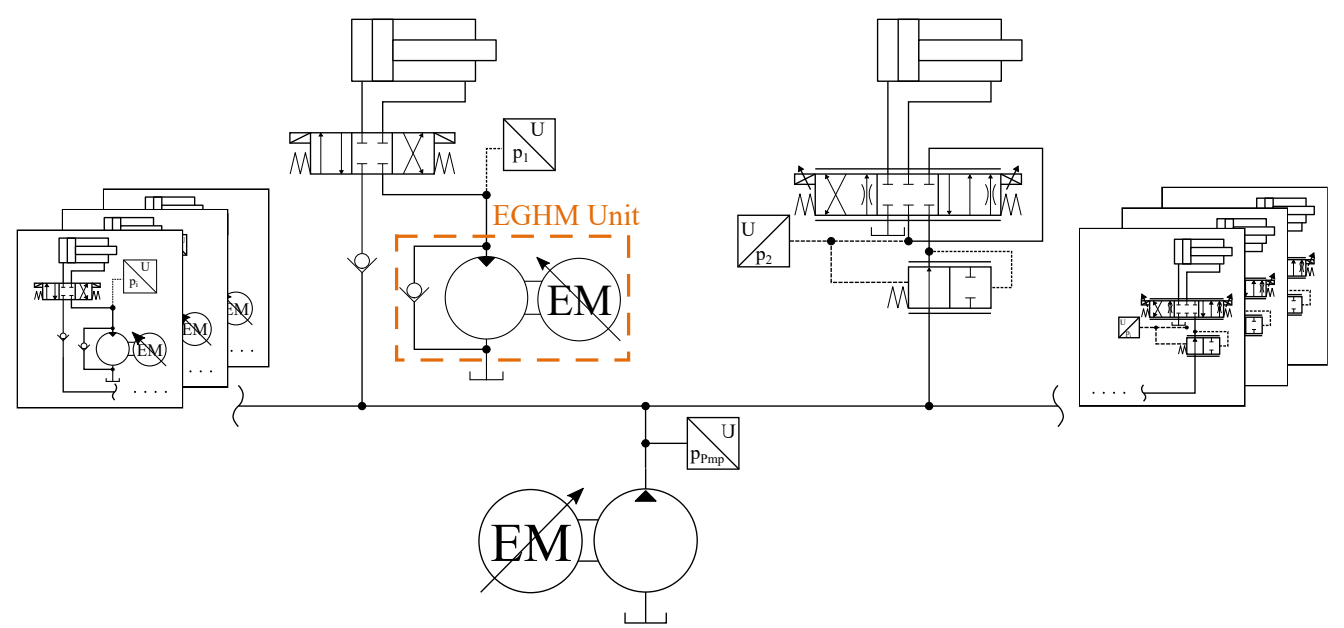

Figure 3. Simplified schematics of the LS system with EGHM units for specific actuators that was presented in [12].

The fixed-cost term $f_{1, E G H M}$ is similar to the conventional LS system since only the attached actuator units differ:

$$
f_{1, E G H M}=9
$$

Similarly, the costs for adding additional low-consuming actuators is low since conventional LS control valves can be used, and the characteristic $c_{2}$ is rated low (1). Only actuators with high energy turnover that utilize an EGHM unit require more-expensive components, and $c_{1}$ is rated 3 . The difference between costs for main actuators and costs for auxiliary actuators (aspects 1 and 2) is made in the algorithm due to this concept where the costs differ between both types.

Large actuator sizes are only slightly more challenging for the EGHM concept than for a conventional LS actuator since the required hydraulic motors and electric machines can get very large. However, if this should be a problem, the concerning actuator can be controlled by a conventional control valve instead; thus, $c_{3}$ is rated 2 . The supply lines are the same as for a conventional LS system; so $c_{4}$ is rated 3 . Power up scaling can be problematic since high pressures can get amplified in this system to even higher pressures. This does not compromise the functionality of the concept, but in order to limit maximum pressures the recuperation potential can be compromised in this case [12]. Thus, $c_{5}$ is rated 2.

If the EGHM-unit is used, the efficiency of an actuator in single operation can be slightly higher than for a conventional LS actuator but is still in a similar range; so $c_{6}$ is rated 3 here as well. $c_{7}$, on the other hand, is reduced to 2 because the EGHM-unit can recuperate the energy that is conventionally throttled due to load-pressure differences [12]. Furthermore, the EGHM units allow recuperation, and $c_{8}$ can generally be rated low. However, as already mentioned, in some operation conditions, pressure limitation measures can limit the recuperation potential, and $c_{8}$ is rated 2 . The characteristics $c_{9}, c_{10}$ and $c_{12}$ are 
rated same as for the conventional LS system due to the similarity. Only $c_{11}$ is increased to 5 since the EGHM units require more space than a conventional valve block would.

$$
\boldsymbol{c}_{E G H M}^{\top}=\left(\begin{array}{llllllllllll}
3 & 1 & 2 & 3 & 2 & 3 & 2 & 2 & 2 & 1 & 5 & 1
\end{array}\right)
$$

\subsection{Electro-Hydraulic Actuators}

In academia, EHAs are one of the most focused concepts for replacing conventional actuator concepts on HDMMs and, therefore, considered here as well. The common principle is using electric variable-speed motors in combination with fixed-displacement pumps in direct connection to a single actuator. This allows to control the pump flow and thus the actuator flow and speed. A detailed review of this actuator concept can be found in [5], where 8 subclasses of EHAs are defined. For this paper, three different types of EHAs are considered for the match evaluation, which can be seen in Figure 4.

a)

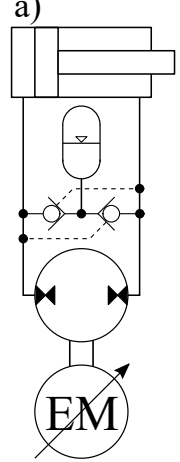

b)

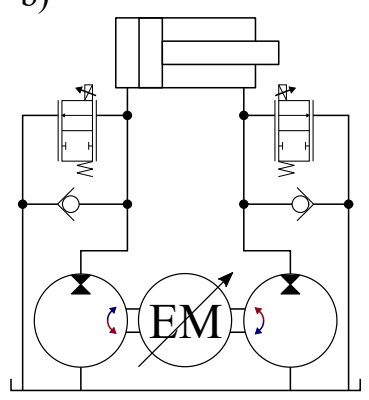

c)

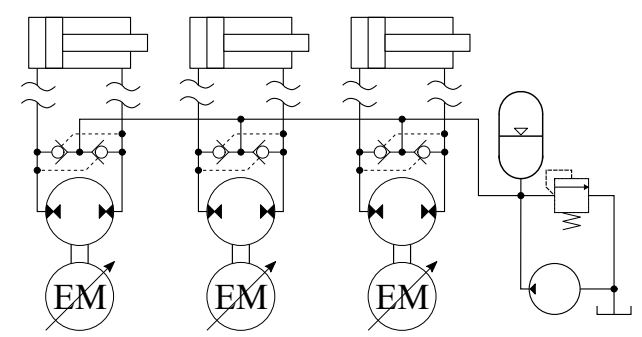

Figure 4. Simplified schematics of considered EHA types (a) compact single pump (b) compact dual pump according to [13] and (c) single-pump centralized.

\subsubsection{Baseline Electro-Hydraulic Actuator: Compact Single-Pump Type}

The single-pump EHA is one of the most common EHA concepts and thus chosen as a baseline EHA here. For the other two EHA concepts, only the differences to this baseline EHA are discussed. Figure 4a shows typical schematics of this type. Due to the unbalanced flow between symmetrical pump and asymmetric cylinder, compensating valves are required. The accumulator provides the pendulum volume and pressure to avoid cavitation.

Because each actuator requires its own electric motor, pump and flow compensation mechanism, the costs for each actuator $c_{1}$ (in Equation (10)) are high (4). Also for auxiliary functions, these costs are high $\left(c_{2}=4\right)$ since the hardware does not change. One the other hand, there are no components that cause fixed costs except for the energy supply which is necessary for each actuator concept anyway, and

$$
f_{1, \text { EHASinglePump }}=3 .
$$

Very large or long actuators are critical for this concept because the pendulum volume is large in these cases as well, and the accumulator size must be rapidly increased [14]. Therefore, $c_{3}$ is rated 4 . The only necessary supply line for these actuators is an electric cable. Because long electric cables are assumed to be significantly less expensive than hydraulic high-pressure lines, $c_{4}$ is only rated 1 .

Power can be scaled up by using larger pumps and motors, which is less problematic. A minor issue can be the lack of flow-compensating valves that can handle higher flows, and two valves in parallel might need to replace a single valve [11]. Thus, $c_{5}$ is rated 2 . Efficiencies of these actuators are relatively high - at least compared to valve-controlled actuators. In [15], hydraulic circuit efficiencies of 75\% could be achieved for different EHAs in experiments. Accordingly, $c_{6}$ is rated 2. Because, the actuators are individual, and each has its own pump, simultaneous actuation has no influence on the efficiency, and $c_{7}$ is 0 . 
Energy can be electrically recuperated, which is only compromised by the hydraulic circuit efficiency and the need for additional electric hardware; therefore, $c_{8}$ is rated 2.

In [16], it was shown that passive cooling of these actuators can be sufficient for low powers (max $1.5 \mathrm{~kW}$ in experiments) and at medium ambient temperature. However, for higher power levels common for HDMMs, active cooling is expected to be necessary. Because the actuators have no hydraulic connection, this requires a cooler for each actuator, which is expensive and results in a high $c_{9}$ value (4).

In the case of compact EHAs, the volume and weight of the actuator not only comprise the hydraulic cylinder but also all other hydraulic components and the electric machine, which are mounted right next to the cylinder. Even though, integrated designs for improved compactness are common, this means that the actuators are much heavier and largertypically in their width, not length-compared to simple hydraulic cylinders. Accordingly, $c_{10}$ is rated 3 and $c_{12} 4$. On the other hand, no centralized hydraulic components are necessary, only a supply of electric energy, and the required space on the main body of the HDMM is small $\left(c_{11}=2\right)$.

$$
\boldsymbol{c}_{\text {EHASinglePump }}^{\top}=\left(\begin{array}{llllllllllll}
4 & 4 & 4 & 1 & 2 & 2 & 0 & 2 & 4 & 3 & 2 & 4
\end{array}\right)
$$

\subsubsection{Compact Dual-Pump Electro-Hydraulic Actuator}

Using two pumps of different size for each actuator allows to compensate the differential cylinder flow with the pumps themselves. The additional valves in Figure $4 \mathrm{~b}$ only manage small flows to avoid cavitation or over-pressurization due to slight mismatches between pump and cylinder ratios [13]. The additional pump might make the EHA slightly more expensive than the single-pump EHA; still, the difference is assumed to be small and $c_{1}$ as well as $c_{2}$ remain 4 . Moreover, no pressurized reservoir is necessary and a vented tank instead of an accumulator can be used for this EHA type. This makes larger actuators less problematic and reduces $c_{3}$ to 1 . At the same time, it might be necessary to install the tank further away from the cylinder because the tank must remain in a stable orientation in order to avoid leakage. Thus, $c_{4}$ is increased to 2 . Scaling up the power is less problematic because only the pumps, not the valves, need to be increased in size $\left(c_{5}=1\right)$. Even though an additional pump is required, it is not assumed that the actuator will be much heavier or larger than the baseline EHA since the pumps could be integrated in one housing and smaller valves as well as a non-pressurized reservoir can be used. Thus, $c_{10}$ an $c_{12}$, same as the other, not mentioned values do not change from the characteristics of the baseline EHA in Section 3.3.1:

$$
\begin{gathered}
\boldsymbol{c}_{\text {EHADualPump }}^{\top}=\left(\begin{array}{cccccccccccc}
4 & 4 & 1 & 2 & 1 & 2 & 0 & 2 & 4 & 3 & 2 & 4
\end{array}\right) \\
f_{1, \text { EHADualPump }}=3
\end{gathered}
$$

\subsubsection{Centralized Electro-Hydraulic Actuator}

Centralizing multiple EHAs by installing the main circuit components on the main body of the HDMM with a common low-pressure supply and long hydraulic lines supplying the actuators, as shown in Figure 4c, has multiple effects on the characteristics compared to the baseline EHA: First, the common low pressure supply increases the fixed costs, and

$$
f_{1, \text { EHACentralized }}=6 \text {, }
$$

but the price per actuator decreases. Thus, $c_{1}$ as well as $c_{2}$ become smaller (3). Furthermore, larger actuators are less problematic because a very large accumulator can still be acceptable when it is mounted on the rear of the vehicle and not next to the cylinder. Thus, $c_{3}$ is reduced to 3 . Moreover, long hydraulic lines across the HDMM are more expensive than electric cables and $c_{4}$ increased to 3 .

The maximum efficiency will decrease because more line losses are present [17] and the charge pump causes losses. This leads to a higher $c_{6}$ (3). Regardless, a further 
benefit is the cooling capability. Instead of using a separate cooler for each EHA, a single cooler can be located in the common low-pressure supply, which is more convenient and reduces $c_{9}$ to 2 .

Moreover, no extra components need to be mounted right next to the cylinder anymore and the volume and mass at the actuator position are rated similar to the valve-controlled concept $\left(c_{10}=1\right.$ and $\left.c_{12}=1\right)$. In return, a lot of volume is required on the main body of the HDMM since multiple pumps and electric machines plus auxiliary systems need to be mounted there $\left(c_{11}=5\right)$. Other, not mentioned characteristics do not change compared to the baseline EHA:

$$
\boldsymbol{c}_{\text {EHACentralized }}^{\top}=\left(\begin{array}{llllllllllll}
3 & 3 & 3 & 3 & 2 & 3 & 0 & 2 & 2 & 1 & 5 & 1
\end{array}\right)
$$

\subsection{Electro-Mechanical Actuators}

At last, an EMA is characterized as a non-hydraulic actuator type. A detailed analysis of EMAs especially for HDMMs can be found in $[18,19]$ in comparison to EHAs, which are the basis of the characterization here. Furthermore, only spindle-type EMAs are considered here.

The price for each single EMA is to be rated even higher than the price for a typical EHA $\left(c_{1}=5\right.$ and $c_{2}=5$ ) because the EMA spindle requires expensive high-precision manufacturing in order to reduce wear of the mechanical components. The fixed cost, on the other hand, are similar to those for EHAs and low:

$$
f_{1, E M A}=3
$$

Modern, large EMAs can reach maximum dynamic forces of multiple hundred kilo Newton which can be sufficient for many medium-sized HDMMs but not to replace very large hydraulic cylinders. Furthermore, long strokes are especially critical for EMAs because of the buckling risk and bending forces that result from the weight of the extended spindle in horizontal positions. Accordingly, $c_{3}$ is rated 3. $c_{4}$ is rated low (1) since only electric cables are required.

It was already mentioned that medium high forces can be reached, but typically, those are only available at low speeds. The reason is the low maximum power limitation of most EMAs which is related to the maximum power of the electric machine and the cooling capability of the spindle. Even though, the efficiency of the spindle and the whole EMA is high $\left(c_{6}=1\right)$ the remaining losses at high powers can be enough to overheat the spindle, which typically has no active cooling capability. For HDMM applications, this is even more critical since environment temperatures can be much higher than the reference temperatures that are given for the maximum power limit in the data sheets. This limits the power-upscaling capability of EMAs $\left(c_{5}=3\right)$ and also shows that cooling is an issue. Active cooling would require a cooler for each actuator, and the spindle would require internal cooling channels, which increases the manufacturing effort even more. Thus, the $c_{9}$ value is rated 5 .

Similar to an EHA, EMAs are self-sustained and simultaneous operation of multiple actuators has no influence on the efficiency $\left(c_{7}=0\right)$. Despite the fact that certain EMA types can be self-locking under certain conditions, which would prevent electric energy recuperation, many EMAs products can be found that are not self-locking-even for high forces-and recuperation is not problematic, especially considering the high spindle efficiency $\left(c_{8}=1\right)$.

Hagen et al. [19] showed for a representative application that EMAs are larger in all dimensions and heavier compared to EHAs with similar force or power, which leads to higher values for $c_{10}$ and $c_{12}$ (4). $c_{11}$ is rated 2, similar to the compact EHAs.

$$
\boldsymbol{c}_{E M A}^{\top}=\left(\begin{array}{llllllllllll}
5 & 5 & 3 & 1 & 4 & 1 & 0 & 1 & 5 & 5 & 2 & 5
\end{array}\right)
$$




\section{Classification of Heavy-Duty Mobile Machine Types}

In this section, the attributes of six typical HDMM types, which are depicted in Figure 5, are analyzed in order to use them for the match evaluation in Section 5. This requires the definition of attribute vectors $\boldsymbol{a}$.

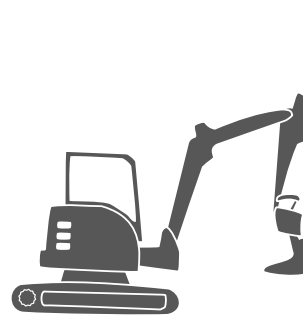

Compact Excavator

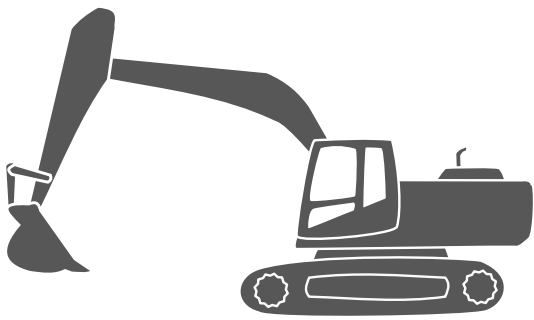

Mid-Size Excavator

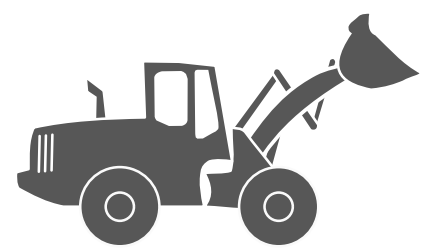

Mid-Size Wheel Loader

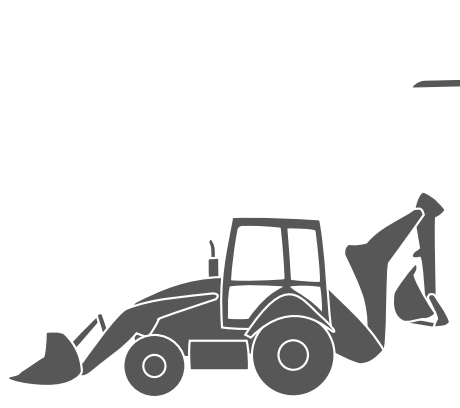

Backhoe Loader

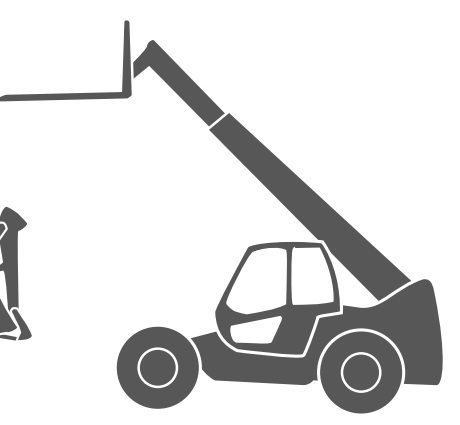

Telehandler

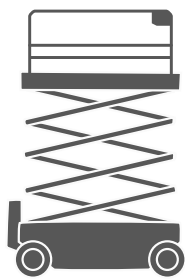

Scissor Lift

Figure 5. Different types of HDMMs that are evaluated in this paper.

\subsection{Compact Excavators}

Excavators are one of the most common HDMMs and their attributes are representative for a number of similar HDMMs. Moreover, the here considered reference machine, Volvo's compact excavator EC15E [20] is a typical representative of a low-power HDMM. Shield, bucket and potential attachments are actuator functions with a rather low energy turnover because they are rarely actuated or see low loads, while boom and stick actuators have higher energy demands; thus, $a_{1}$ is rated 2 , and $a_{2}$ is rated 3 . Due to the compact size of the machine, the actuators are rather small as well, and $a_{3}$ is rated 1 . Furthermore, most actuators are located close to the main vehicle body, but the bucket actuator is mounted at the end of the relatively long excavator arm. Accordingly, $a_{4}$ is chosen to be 2 .

On the exemplary excavator, each actuator can be supplied with up to $10 \mathrm{~kW}$ of hydraulic power [20], which is relatively low; thus, $a_{5}$ is rated 2. Moreover, typical excavator tasks, such as truck loading, digging or grading, involve mainly the implement actuators and less driving. As a result, the attribute $a_{6}$, representing the relative energy consumption of the implements, is rated high (5). Furthermore, analyses of work cycles show that simultaneous actuation of multiple work functions is common especially for expert operators; thus, $a_{7}$ is rated 5 .

The amount of load braking, which can be used for recuperation, always depends on the work task. Grading does not offer a lot of recuperation potential, but most other tasks are cyclic and involve up and down movement. Down movement can be used for recuperation, but typically the boom is lifted with load, the load is dumped and then the boom is lowered empty, which offers less potential energy. Still, the potential $a_{8}$ is rated high (4), but could be adjusted if a specific work cycle is supposed to be focused for the match evaluation. The attribute $a_{9}$ can also only be specified if a concrete application is known and is thus rated with a neutral/medium value of 3 here.

Since the actuator arm is not surrounded by other machine parts, there is relatively much installation space for the actuators; still, the installation lengths are limited by the kinematic chains and $a_{10}$ is rated 2 . On the main body, space is limited but can be 
made available by replacing counterbalance weights with drive train components; so $a_{11}$ is rated 2. Moreover, the length of the excavator arm leads to significant influences of the mass of bucket and stick actuators on the boom actuator and thus the payload. This was demonstrated in [21], and $a_{12}$ is rated 4 accordingly. The resulting attribute vector is

$$
\boldsymbol{a}_{\text {compactExcavator }}=\left(\begin{array}{llllllllllll}
2 & 3 & 1 & 2 & 2 & 5 & 5 & 4 & 3 & 2 & 2 & 4
\end{array}\right) .
$$

\subsection{Mid-Size Excavators}

A mid-size excavator is included here in contrast to the compact excavator in order to point out the differences between similar machines of different sizes. Most attributes can be adopted from the compact excavator but a few change:

The actuator size increases significantly and maximum forces of several hundred kilo Newton are not uncommon. Thus, $a_{3}$ as well as $a_{5}$ are increased to $4 . a_{4}$ is increased to 3 since the arm length is increased. The resulting attribute vector is

$$
\boldsymbol{a}_{\text {midSizeExcavator }}=\left(\begin{array}{llllllllllll}
2 & 3 & 4 & 3 & 4 & 5 & 5 & 4 & 3 & 2 & 2 & 4
\end{array}\right) .
$$

\subsection{Mid-Size Wheel Loader}

The actuated implement parts of wheel loaders, such as the here considered Volvo LH90 [22], typically comprise boom, shovel, steering and sometimes an additional attachment. While boom and shovel can have high energy turnover, the other functions typically do not. Accordingly, there are a few actuators of each type (low/high energy turnover), and $a_{1}$ as well as $a_{2}$ are rated 2 .

Especially the tilt cylinders can be large in diameter (cylinder bore of $180 \mathrm{~mm}$ at a stroke of $430 \mathrm{~mm}$ [22]); thus, $a_{3}$ is rated 3. Because the boom is rather short, all actuators are relatively close to each other and the main vehicle body, which leads to a low $a_{4}$ (2) and also a low influence of the actuator mass on the payload $\left(a_{12}=2\right)$. On the considered conventional wheel loader [22], a single actuator can be supplied with up to $88 \mathrm{~kW}$ of hydraulic power, which is considered high and leads to a high $a_{5}$ (4). Because common wheel loader work cycles like truck loading or Y-cycles involve significant amounts of driving, $a_{6}$ is only rated medium (3). Simultaneous operation of boom and shovel cylinders is common but not necessarily constantly the case, which leads to $a_{7}$ being 3 . Lowering the boom involves braking against gravity, which can be used for recuperation, but the boom is most often lowered empty, and lowering makes up only a short part of common work cycles, which involve more driving instead. Accordingly, $a_{8}$ is rated 3. $a_{9}$ is rated neutral (3) because the specific application environment is not known.

Similarly to an excavator arm, the wheel loader boom is relatively exposed; so mounting larger actuators should be less problematic $\left(a_{10}=2\right)$, and also the wheel-loader main body offers space on the rare, potentially by replacing the counter weight $\left(a_{11}=2\right)$. The resulting attribute vector is

$$
\boldsymbol{a}_{\text {wheelLoader }}=\left(\begin{array}{llllllllllll}
2 & 2 & 3 & 2 & 4 & 3 & 3 & 3 & 3 & 2 & 2 & 2
\end{array}\right) .
$$

\subsection{Backhoe Loader}

Backhoes, such as the Case 580 Super N [23] considered here, combine a lot of different implement functions. The loader function and excavator function cannot be used at the same time and are therefore frequently in standby. Furthermore, many auxiliary functions require cylinder actuators but are only used once in a while: side supports, boom swing, excavator telescope arm, bucket gripper and others. All in all, this means a high number of actuators with low energy turnover and only a few with a medium high turnover $\left(a_{1}=2\right.$ and $\left.a_{2}=4\right)$.

Because backhoes are rather compact, actuator sizes are medium or small (more than half of the cylinders have bore diameters smaller than $100 \mathrm{~mm}$ [23]) and $a_{3}$ is rated 2 . Furthermore, actuators are located close to the main body $\left(a_{4}=2\right)$. Moreover, up to $65 \mathrm{~kW}$ 
of hydraulic power is available but can most likely not be used for the small actuators entirely, and $a_{5}$ is rated 3. No specific work cycle is considered here because backhoes are too versatile; however, driving is one of the main functions. Accordingly, $a_{6}$ is rated medium (3). Actuation of more than one actuator at the same time is also common for backhoes, and $a_{6}$ is rated 3 .

The main implement parts, excavator boom and loader boom, move vertically; thus, there is a potential for energy recuperation, but the masses are commonly rather low. Therefore, $a_{8}$ is rated 3. $a_{9}$ is rated neutral (3) because the specific application environment is not known.

The space on a backhoe is generally limited. Because several actuators are distributed around the whole machine, there is neither much space next to the actuators $\left(a_{10}=4\right)$ nor on the main body of the machine $\left(a_{11}=4\right)$. However, the actuator-mass influence can be rated low due to the short distances between actuator locations and main body $\left(a_{12}=2\right)$. The resulting attribute vector is

$$
\boldsymbol{a}_{\text {backhoe }}=\left(\begin{array}{llllllllllll}
2 & 4 & 2 & 2 & 3 & 3 & 3 & 3 & 3 & 4 & 4 & 2
\end{array}\right) .
$$

\subsection{Telehandler}

Telehandlers represent another type of HDMMs, which is characterized by long-stroke actuators. The standard implement actuators of telehandlers such as the MHT 10130 [24], which is considered for this analysis, are for steering, boom actuation, tool tilting, telescope extension but also frame leveling, stabilizers or attachments. It can be assumed that boom, telescope and tilt functions have a relatively high energy turnover, while the other functions do not; accordingly, $a_{1}$ as well as $a_{2}$ are rated 3 .

Characteristic is the telescopic actuator, which is large in volume due to the long stroke of more than $7 \mathrm{~m} \mathrm{[24].} \mathrm{Accordingly,} a_{3}$ is rated 5. Furthermore, the tilt actuator, which is mounted on the extending part, requires a long and flexible connection to the main part of the vehicle, which results in a high $a_{4}$ (5). Moreover, the actuators can be supplied with $90 \mathrm{~kW}$ of hydraulic power, which is significant and leads to a high $a_{5}$ (4). Similarly to wheel loaders, telehandler work cycles typically involve a significant amount of driving, and $a_{6}$ is rated medium (3). Simultaneous operation of multiple actuators is common for telehandlers as well but not necessarily constantly the case $\left(a_{7}=3\right)$.

The load braking and thus recuperation potential of telehandlers can be considered high because they are often equipped with forks to handle pallets or other compact objects. These objects typically cannot be dumped, and the telehandler must lower the heavy objects again. Compared to the empty lowering typical for HDMMs with bucket or shovel, the potential energy and recuperation potential are much higher in this case, and $a_{8}$ is rated 4. $a_{9}$ is rated neutral (3) because the specific application environment is not known.

Since many actuators are mounted within mechanical structures, the available space next to the actuators is limited $\left(a_{10}=3\right)$ as well as the space on the main body $\left(a_{11}=3\right)$. Also, high actuator masses are critical since the tilt actuator mass for example can have a significant influence on the boom actuator at longer telescope extension lengths $\left(a_{12}=4\right)$. The resulting attribute vector is

$$
\boldsymbol{a}_{\text {telehandler }}=\left(\begin{array}{llllllllllll}
3 & 3 & 5 & 5 & 4 & 3 & 3 & 4 & 3 & 3 & 3 & 4
\end{array}\right) .
$$

\subsection{Scissor Lift}

Finally, a scissor lift is included as a typical representative of the class of aerial work platforms. Many scissor lifts comprise only one linear actuator for lifting (some larger ones include also two) and additional actuators for steering with relatively low energy turnover $\left(a_{1}=1\right.$ and $\left.a_{2}=2\right)$. The size and maximum force of the cylinders is typically low as the maximum work load of the platform is not more than a few people plus working equipment; so $a_{3}$ is rated 2. Furthermore, the actuator speed is typically low, since humans stand on the moving part. Accordingly the required actuator power is low as well $\left(a_{5}=1\right)$. 
The actuator is usually mounted right to the carriage so there is no long distance for actuator supply lines, and $a_{4}=1$. The relative amount of energy that is spend for the linear actuators compared to driving hardly depends on the work cycle and is thus rated medium here as default $\left(a_{6}=3\right)$. Since most scissor lifts have only one lifting actuator, there is no chance of simultaneous operation, only for the exception of scissor lifts with two actuators; so $a_{7}$ is rated 1. The amount of load braking is quit high since scissor lifts move solely vertically and with the same load down as up, but the load is relatively low compared to loss terms in the mechanism. Accordingly, $a_{8}$ is rated 4. Again, the environment temperatures depend on the specific application, but there is a tendency that scissor lifts are operated indoors where it is colder. Hot areas such as deserts or tropics are less likely, and $a_{9}$ is rated 2. Fully retracted, the lift needs to be very compact and there is not much space at the actual actuator position nor at the main body, and $a_{10}$ as well as $a_{11}$ are rated 3. Finally, the influence of the actuator mass is assumed to be very low since the actuator is only mounted to the lifting parts if two actuators are used $\left(a_{12}=1\right)$. The resulting attribute vector is

$$
a_{\text {scissorLift }}=\left(\begin{array}{llllllllllll}
1 & 2 & 2 & 1 & 1 & 3 & 1 & 4 & 2 & 3 & 3 & 1
\end{array}\right) .
$$

\section{Evaluation of Exemplary Matches and Discussion}

For this section, 36 matches have been formed out of the six actuator types analyzed in Section 3 and the six HDMM types investigated in Section 4. The actuator-HDMM matches have been evaluated three times according to Equation (3) with different weight matrices for different evaluation focuses. The three tables for each evaluation are now analyzed in order to get insights about the match qualities and to make general statements about HDMM-actuator matches as well as the algorithm.

Marked in green, the best matches for each HDMM type can be seen in Table 2 for the default weight matrix $W_{0}$. In this scenario, the algorithm indicates that the doublepump EHA is most suitable for most HDMMs due to achieving the lowest mismatch values. However, for the compact excavator, the centralized EHA is ruling, while a backhoe might also generally match with the EGHM-LS concept. These outcomes are in line with the common knowledge that EHAs have the strength of high energy-efficient and that the dual-pump concept can be scaled up easily in size and power. Also, the suitability of the LS concept for the backhoe can be explained by the fact that it has a high number of actuators; so saving costs per actuators by using simple control valves for auxiliary drives seems to be effective.

Furthermore, several concepts achieve mismatch values for the same machine that are very close together. In these cases, it cannot be said which concept is indeed more suitable for the HDMM than another because the evaluation algorithm is rather sensitive towards imprecise analyses of the characteristics and attribute vectors. Still, better-worse decisions can be made whenever the mismatch-value differences are large.

Table 2. Mismatch values for all 36 actuator-HDMM matches and weight matrix $\boldsymbol{W}=\boldsymbol{W}_{0}$. The best values for each HDMM type have been marked green.

\begin{tabular}{|c|c|c|c|c|c|c|}
\hline & $\begin{array}{l}\text { LS with Valve } \\
\text { Control Only }\end{array}$ & $\begin{array}{l}\text { LS with Valve } \\
\text { or EGHM } \\
\text { Control }\end{array}$ & $\begin{array}{c}\text { EHA Single } \\
\text { Pump }\end{array}$ & $\begin{array}{l}\text { EHA Dual } \\
\text { Pump }\end{array}$ & $\begin{array}{c}\text { EHA } \\
\text { Centralized }\end{array}$ & EMA \\
\hline compact excavator & 83 & 74 & 75 & 71 & 68 & 76 \\
\hline mid-size excavator & 89.5 & 85.5 & 91.5 & 77 & 82.5 & 91.5 \\
\hline wheel loader & 68 & 68 & 73 & 61 & 66 & 76 \\
\hline backhoe & 73 & 72 & 80 & 72 & 73 & 86 \\
\hline telehandler & 85 & 86.5 & 99 & 82.5 & 88.5 & 101.5 \\
\hline scissor lift & 60.5 & 54 & 57 & 52.5 & 55 & 55.5 \\
\hline
\end{tabular}


For example, the purely valve-controlled LS concept and the EMA achieve mismatch values that are much higher than the best value for most of the HDMMs. Normal reasoning could lead to the explanation that the LS concept performs worse due to its low efficiency despite its low costs per actuator. Furthermore, it could be reasoned that the EMA, vice versa, performs worse due to the high component costs despite its high energy efficiency. In order to validate if the evaluation algorithm conforms with these ideas, the other two evaluation results with cost and with efficiency focus, respectively, are analyzed.

The investment-costs-focused evaluation results in Table 3 show that now the LS concepts, especially the purely valve-controlled one, are performing better or even outperform the EHA solutions for the backhoe and telehandler, which confirms the ideas in the last paragraph—also by the fact that the EMA performs even worse now.

Table 3. Mismatch values for all 36 actuator-HDMM matches and weight matrix $\boldsymbol{W}=\boldsymbol{W}_{0} \cdot \boldsymbol{W}_{\text {costs. }}$ The best values for each HDMM type have been marked green.

\begin{tabular}{|c|c|c|c|c|c|c|}
\hline & $\begin{array}{l}\text { LS with Valve } \\
\text { Control Only }\end{array}$ & $\begin{array}{l}\text { LS with Valve } \\
\text { or EGHM } \\
\text { Control }\end{array}$ & $\begin{array}{l}\text { EHA Single } \\
\text { Pump }\end{array}$ & $\begin{array}{l}\text { EHA Dual } \\
\text { Pump }\end{array}$ & $\begin{array}{c}\text { EHA } \\
\text { Centralized }\end{array}$ & EMA \\
\hline compact excavator & 100 & 95 & 99 & 96 & 92 & 105 \\
\hline mid-size excavator & 108 & 108 & 116 & 103 & 108 & 121 \\
\hline wheel loader & 84 & 88 & 93 & 82 & 87 & 100 \\
\hline backhoe & 91 & 94 & 108 & 101 & 100 & 120 \\
\hline telehandler & 107.5 & 115 & 128.5 & 114.5 & 120 & 137 \\
\hline scissor lift & 74 & 69.5 & 66.5 & 70.5 & 71.5 & 74 \\
\hline
\end{tabular}

Furthermore, it is conspicuous that the scissor lift achieves mismatch values that are very close together for each actuator concept. This indicates that this HDMM is more open towards applying a novel actuator concept from a cost perspective compared to others. In order to choose which concept to prefer for the scissor lift, another aspect must be considered-e.g., the efficiency which is focused in the evaluation presented in Table 4:

Table 4. Mismatch values for all 36 actuator-HDMM matches and weight matrix $W=W_{0} \cdot W_{\text {efficiency }}$. The best values for each HDMM type have been marked green.

\begin{tabular}{|c|c|c|c|c|c|c|}
\hline & $\begin{array}{l}\text { LS with Valve } \\
\text { Control Only }\end{array}$ & $\begin{array}{l}\text { LS with Valve } \\
\text { or EGHM } \\
\text { Control }\end{array}$ & $\begin{array}{c}\text { EHA Single } \\
\text { Pump }\end{array}$ & $\begin{array}{l}\text { EHA Dual } \\
\text { Pump }\end{array}$ & $\begin{array}{c}\text { EHA } \\
\text { Centralized }\end{array}$ & EMA \\
\hline compact excavator & 133 & 107 & 93 & 89 & 91 & 85 \\
\hline mid-size excavator & 139.5 & 118.5 & 109.5 & 95 & 105.5 & 100.5 \\
\hline wheel loader & 101 & 89 & 85 & 73 & 81 & 82 \\
\hline backhoe & 106 & 93 & 92 & 84 & 88 & 92 \\
\hline telehandler & 123 & 109.5 & 113 & 96.5 & 105.5 & 108.5 \\
\hline scissor lift & 92.5 & 73 & 71 & 64.5 & 72 & 62.5 \\
\hline
\end{tabular}

With an efficiency focus, the EMA is winning for the scissor lift and also the compact excavator. In this point, the evaluation algorithm is also confirmed by the fact that a scissor lift is, to the authors' knowledge, the first commercially available, fully electric HDMM that utilizes EMAs where previously hydraulic cylinders have been used [25]. For the other HDMMs, the EMA concept performs better as well, but the dual-pump EHA still achieves the best values. The valve-controlled LS concept achieves the worst values in this evaluation which is again in line with the reasoning in the previous paragraph and confirms the practicality of the match evaluation algorithm. 


\section{Limitations of the Evaluation Concept}

For the interpretation of the mismatch values that result from the evaluation algorithm, it is important to consider that the evaluation concept is based on certain assumptions and that this leads to a few limitations. Those limitations are explained in this section alongside ways of how to address them.

An essential idea of the concept is that each HDMM can only be equipped with one type of actuators. This might be reasonable since scaling effects apply when multiple similar actuators are used and the costs generally decrease, but applying two different actuator concepts on the same machine is still feasible. For example, valve-controlled centralized actuators could be used for the auxiliary functions of an HDMM while the main actuators utilize EHAs. However, the algorithm is also capable of covering this case by handling the combination of two actuator concepts as an additional separate "actuator concept".

Another assumption is the idea that generally all actuator concepts are compatible with every HDMM. The problem aspects in the algorithm cannot indicate weather a combination is feasible at all or not. A $400 \mathrm{t}$ mining excavator, for example, can hardly be equipped with EMAs that are available nowadays-Hagen et al. pointed out that the maximum continuous power output of modern EMAs is in the range of a few kilo Watts [18]. Accordingly, the algorithm should only be used for the evaluation of matches that are known to be generally feasible.

A further step for improving the quality of the results is to include even more problem aspects, which would increase the number of dimensions considered in the evaluation process. However, this can only be beneficial if the weight factors for each aspect are chosen wisely, so that no aspect obtains more influence on the evaluation results than it should. A lot of potential lays in the fine tuning of the weight factors as well as the attribute and characteristics vectors. It should be noticed that in this paper all evaluation parameters have been rated by the authors only, with a few data points and mainly personal knowledge. In order to increase the objectivity, multiple engineers should do the rating and validate each other's results if possible.

\section{Conclusions}

A novel algorithm that is capable of quickly and systematically evaluating the qualities of a large set of potential matches between linear actuators and HDMM applications was proposed in this paper. For each actuator-machine combination, a mismatch value is calculated, which can indicate that an actuator concept is more suitable for a specific HDMM than another concept that received a higher mismatch value-or vice versa, a specific HDMM is more compatible with a certain actuator concept than another HDMM if it achieves a lower mismatch value. For the preparation of the evaluation, each actuator concept and each HDMM of interest must simply be analysed concerning general problem aspects that can possibly apply to an actuator-machine combination. This way, the evaluation concept is highly modular and allows to include additional actuator concepts or HDMM types in the evaluation at a later point. This makes the algorithm especially useful for less-experienced engineers who can apply the algorithm to new HDMMs without having deep knowledge about the pre-analysed actuator concepts, but also experienced engineers can utilize the algorithm to quickly analyse the influence of specific evaluation parameters on different HDMM-actuator combinations for example.

For a demonstration, 36 matches between six different actuator concepts and six HDMM types have been evaluated with different emphases: a general evaluation, component costs, or energy-efficiency. The results of the evaluation algorithm are generally in line with direct reasoning, which confirms the functionality of the algorithm. Findings are that EHA concepts, especially with dual-pump design, perform especially well. However, conventional LS systems can rule for certain HDMM types as well and in general if low component costs are focused. Furthermore, a potential for EMAs could be identified if energy efficiency is focused and if the HDMM shows specific attributes, such as low number of actuators or low power level. 
Moreover, remaining limitations of the concept were analyzed and confirmed the initial thought that this evaluation algorithm is only capable of pointing out main tendencies in the compatibility between different actuator concepts and HDMM applications. The algorithm is not intended to replace conventional requirement and condition management as well as simulation studies for the detailed evaluation of a specific HDMM system configuration. However, the algorithm can be a powerful tool for the first, easy and quick evaluation of a large solution space of potential actuator-machine matches, and improving its performance by involving more engineers in the parameter identification process should be targeted for the future.

Author Contributions: Conceptualization, D.F. and T.M.; methodology, D.F.; formal analysis, D.F.; investigation, D.F.; writing — original draft preparation, D.F.; writing — review and editing, D.F. and T.M.; visualization, D.F.; and supervision, T.M. All authors have read and agreed to the published version of the manuscript.

Funding: This project received funding from the European Union's Horizon 2020 research and innovation programme under the Marie Skłodowska-Curie grant agreement No. 858101.

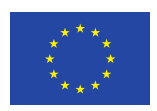

Conflicts of Interest: The authors declare no conflict of interest.

\begin{tabular}{|c|c|}
\hline \multicolumn{2}{|c|}{ Abbreviations } \\
\hline \multicolumn{2}{|c|}{ The following abbreviations are used in this manuscript: } \\
\hline EGHM & electric generator and hydraulic motor \\
\hline EHA & electro-hydraulic actuator \\
\hline EMA & electromechanical actuator \\
\hline HDMM & heavy-duty mobile machine \\
\hline & load sensing \\
\hline OR & operations research \\
\hline
\end{tabular}

\section{References}

1. Padovani, D.; Rundo, M.; Altare, G. The Working Hydraulics of Valve-Controlled Mobile Machines: Classification and Review. J. Dyn. Syst. Meas. Control 2020, 142. [CrossRef]

2. Weber, J. Independent metering systems. Int. J. Hydromechatron. 2018, 1, 91-106. [CrossRef]

3. Optimal Control and Energy-Saving Anaysis of Common Pressure Rail Architectures: HHEA \& STEAM. In Proceedings of the BATH/ASME 2020 Symposium on Fluid Power and Motion Control, Online, 9-11 September 2020.

4. Rahmfeld, R.; Ivantysynova, M.; Weber, J. Displacement Controlled Wheel Loader-A simple and clever Solution. In Proceedings of the 4th, International Fluid Power Conference, Dresden, Germany, 25-26 March 2004; pp. 183-196.

5. Ketelsen, S.; Padovani, D.; Andersen, T.; Ebbesen, M.; Schmidt, L. Classification and Review of Pump-Controlled Differential Cylinder Drives. Energies 2019, 12, 1293. [CrossRef]

6. Vael, G.; Achten, P.; Potma, J. Cylinder Control with the Floating Cup Hydraulic Transformer. In Proceedings of the Eighth Scandinavian Conference on Fluid Power, Finland, Tampere, 7-9 May 2003; pp. 175-190.

7. Linjama, M. Digital Fluid Power-State of the Art. In Proceedings of the 12th Scandinavian International Conference on Fluid Power, Tampere, Finland, 18-20 May 2011.

8. Thomson Industries Linear Actuators for On/Off-Highway Vehicles CPEN-0005-01A | 20161202TJ, 2016. Available online: https:/ / www.thomsonlinear.com/downloads/capabilities/Linear_Actuators_for_OnOff_Highway_Vehicles_cpen.pdf (accessed on 21 October 2020).

9. Ritchey, T. Wicked Problems. Acta Morphol. Gen. 2013, 2.

10. Fassbender, D.; Minav, T. Finding the Perfect Match: Different Heavy-Duty Mobile Applications Call for Different Actuators. In Proceedings of the First International Electronic Conference on Actuator Technology: Materials, Devices and Applications, Online, 23-27 November 2020. [CrossRef]

11. Schmidt, L.; Ketelsen, S.; Brask, M.H.; Mortensen, K.A. A Class of Energy Efficient Self-Contained Electro-Hydraulic Drives with Self-Locking Capability. Energies 2019, 12, 1866. [CrossRef]

12. Fassbender, D.; Minav, T.; Brach, C.; Kalevi, H. Improving the Energy Efficiency of Single Actuators with High Energy Consumption: An Electro-Hydraulic Extension of Conventional Multi-Actuator Load-Sensing Systems. In Proceedings of the 17th Scandinavian International Conference on Fluid Power, Linköping, Sweden, 1-2 June 2021. 
13. Pedersen, H.C.; Schmidt, L.; Andersen, T.O.; Brask, M.H. Investigation of New Servo Drive Concept Utilizing Two Fixed Displacement Units. JFPS Int. J. Fluid Power Syst. 2014, 8. [CrossRef]

14. Ketelsen, S.; Kolks, G.; Andersen, T.O.; Schmidt, L.; Weber, J. Bootstrap reservoir concepts for electro-hydraulic compact cylinder drives. In Proceedings of the 12th International Fluid Power Conference, Dresden, Germany, 9-11 March 2020; Volume 3, pp. 201-215. [CrossRef]

15. Michel, S.; Weber, J. Energy-efficient electrohydraulic compact drives for low power applications. In Fluid Power and Motion Control 2012; University of Bath: Bath, UK, 2012; pp. 94-108.

16. Michel, S.; Weber, J. Prediction of the thermo-energetic behaviour of an electrohydraulic compact drive. In Proceedings of the 10th International Fluid Power Conference, Dresden, Germany 8-10 March 2016; pp. 219-233.

17. Casoli, P.; Scolari, F.; Minav, T.; Rundo, M. Comparative Energy Analysis of a Load Sensing System and a Zonal Hydraulics for a 9-Tonne Excavator. Actuators 2020, 9, 39. [CrossRef]

18. Hagen, D.; Pawlus, W.; Ebbesen, M.K.; Andersen, T.O. Feasibility Study of Electromechanical Cylinder Drivetrain for Offshore Mechatronic Systems. Model. Identif. Control Nor. Res. Bull. 2017, 38, 59-77. [CrossRef]

19. Hagen, D.; Padovani, D.; Choux, M. Guidelines to Select Between Self-Contained Electro-Hydraulic and ElectroMechanical Cylinders. In Proceedings of the 15th IEEE Conference on Industrial Electronics and Applications (ICIEA2020), Kristiansand, Norway, 9-13 November 2020.

20. Volvo-Construction-Equipment. EC15E Compact Excavator Data Sheet. Available online: https://www.volvoce.com/-/ media/volvoce/global/products/excavators /compact-excavators/brochures/brochure_ec15e_t4f_en_21_20056711_b.pdf?v= DDZCPw (accessed on 29 October 2020).

21. Niraula, A.; Zhang, S.; Minav, T.; Pietola, M. Effect of Zonal Hydraulics on Energy Consumption and Boom Structure of a Micro-Excavator. Energies 2018, 11, 2088. [CrossRef]

22. Volvo-Construction-Equipment. Wheel Loaders L60H, L70H, L90H. Available online: https://www.volvoce.com/- / media/ volvoce/global/products /wheel-loaders/wheel-loaders/brochures/brochure_160h_170h_190h_t4f_en_22_voe2240009459.pdf? $\mathrm{v}=\mathrm{ngRPPw}$ (accessed on 28 October 2020).

23. Case-Construction. 580SN Backhoe Loader Data Sheet. Available online: https://assets.cnhindustrial.com/casece/nafta/assets/ Brochures/Products/Backhoe-Loaders/580-Super-N/BL-580SN-Specs-201810.pdf (accessed on 29 October 2020).

24. Manitou-BF-SA. MHT 10130—Telehandler Data Sheet. Available online: https://www.manitou.com/en/p/VOrvHiwAAC4 AxE85 (accessed on 28 October 2020).

25. JLG Industries Inc. AE1932 DaVinci All-Electric Scissor Lift-Specification Sheet. Available online: https://www.jlg.com/en/ equipment/scissor-lifts/electric/davinci-series-scissor-lifts/ae1932 (accessed on 14 April 2021). 